\title{
Asia Syndrom: Diagnosis and Surgical Approach
}

Guerrissi J O*

Department of Plastic and Reconstructive Surgery, Dr. Cosme Argerich Hospital, Argentina

*Corresponding author: Guerrissi J O, Department of Plastic and Reconstructive Surgery, Dr. Cosme Argerich Hospital, Argentina

Received: September 14, 2016; Accepted: January 03, 2017; Published: J anuary 09, 2017

\section{Abstract}

The use of silicone implants for breast aesthetic and reconstructive surgery has been the method most widely used since the mid-twentieth century and every day has more followers both by patients and plastic surgeons.

Its implementation as biologically inert material has been reviewed following the report of cases linked to autoimmunity collectively known as auto inflammatory syndrome adjuvants. While the low incidence of reported cases currently prevents the establishment of parameters that contraindicate the use of silicone there is a predisposition to relate with the onset of autoimmune diseases known or not, including genetic predisposition to them.

However, there are publications of different authors that associate clinical pictures as the so-called Asian syndrome or a variety of Still's disease, with the presence of silicone breast implants.

The reason for this presentation is to draw attention to the possibility of the onset of disease through immunological mechanisms associated with the presence of silicone in the body, generating not only a difficult differential diagnosis and effective treatment also.

Keywords: Still's disease; Thorax CT; Implant surgery; Diagnosis

\section{Introduction}

The utilization of silicone implants for esthetic and reconstructive breast surgery has been the most spread method since middle XX century and it has each day more followers as much as from patients as from plastic surgeons.

Its implementation as a biologically inert material has been reviewed since the report of cases linked with autoimmunity known collectively as "Auto inflammatory syndrome induced by adjuvants". Although the low incidence of cases at the moment prevents us from setting up parameters which contraindicate the use of silicone, there is an inclination to relate it with the appearance of known or unknown autoimmune diseases, including the genetic predisposition.

However, there are publications from different authors [1-4] which associate clinical profiles like the denominated ASIA syndrome or a variety of still disease with the presence of silicone implants.

The reason of this presentation is to attract attention about the possibility of appearance of disease through immunological mechanisms associated to the presence of silicone in the human body, generating not only a difficult differential diagnosis but also an efficient treatment application.

\section{Materials and Methodology}

A 61 year old female patient consults to clinical specialty presenting a 10 months evolution fever associated to myalgia, arthralgia and enthesitis.

The patient relates as part of her medical background the inclusion of breast implants in 1987 and a replacement in 2005 due to the rupture of one of the prosthesis. The physical examination reveals a $4 \mathrm{x} 4 \mathrm{~cm}$ underarm lymphadenophatie, tender-stony hard consistency, movable and painful at palpation (Figure 1).

In both upper and lower limbs and thorax several papules had been detected, either isolated or group together, but without any clinical manifestation (Figure 2).

In laboratory practices that include acute phase reactants, immunity studies and hormonal profile, the following parameters had been verified: elevation of acute phase reactants, leukocytosis with lymphocytary predominance, mycrocitic anemia and elevation of hepatic enzymes.

The evaluation of autoimmune disorders was negative the same as the bacteriological and viral exams. The only positive discovery was a past infection by cytomegalovirus and Epstein Barr virus (Table 1).

The imaging studies by computed tomography of brain, thorax, abdomen and pelvis with and without intravenous and oral contrast, video endoscopy of the high and low digestive tract, abdominal and gynecological echography, mammography and echocardiogram, revealed: 1) Bilateral cervical, supraclavicular and underarm adenopathies not larger than $1 \mathrm{~cm}$. 2) Bilateral pleural effusion with bibasal collapsed lung and laminar pericardial effusion as well. 3) Homogenous splenomegaly measuring $157 \mathrm{~mm}$. 4) Hypodense image in VII segment of the liver measuring $4 \mathrm{~mm}$ with a cystic aspect. 5) Breast implants without any signs of rupture. In left breast an anechoic image measuring $28 \times 6 \mathrm{~mm}$ can be noticed (Figure 3 ).

The surgical decision was the bilateral removal of the breast implants and the capsules. In the upper external quadrant of the left breast, three nodular lesions which spread to the underarm are removed (Figures 4 and 5).

The histological analysis informed siliconomas (nonspecific granulomatous lesions organized around liquid silicone). The flow 


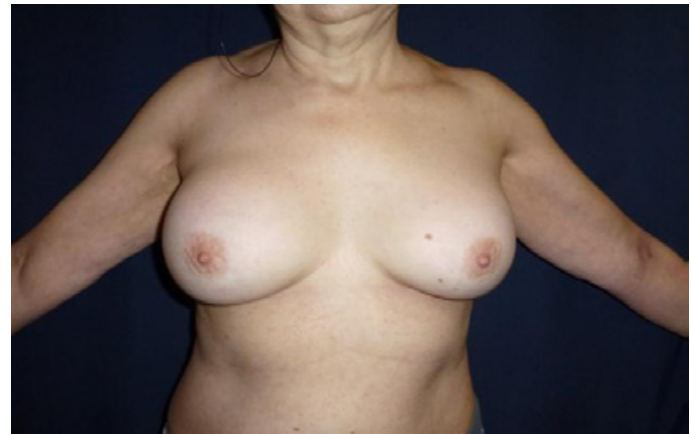

Figure 1: Breast implant. Slight asymmetry with right breast predominance.

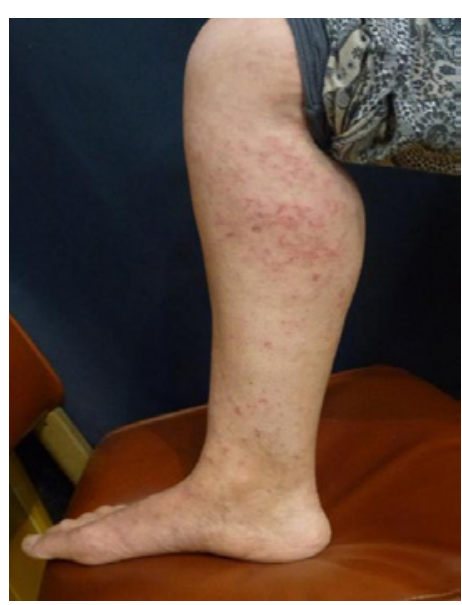

Figure 2: Isolated papules distributed in all four limbs and chest.

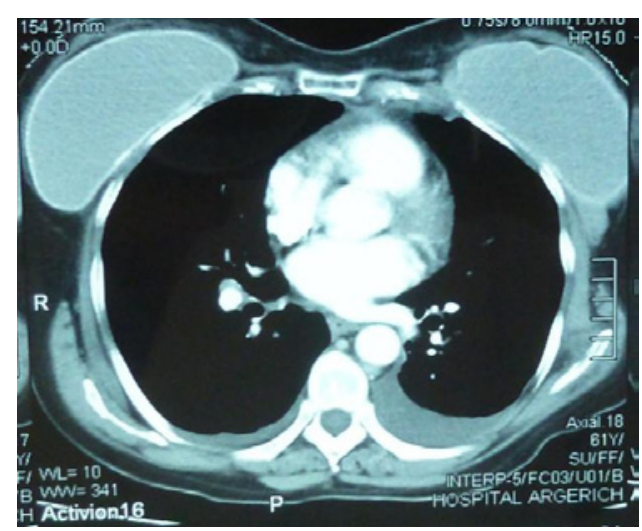

Figure 3: CT chest, thickening of soft left chest.

citometry result was negative.

In the postoperative period the patient received daily doses of meprednisone $20 \mathrm{mg} /$ day and progressed with remission of the febrile records, joint and muscular symptoms and gradual decline of the acute phase reactants in the laboratory analysis, which allowed the doctors to gradually decrease the glucocorticoids dose.

\section{Discussion}

The persistent fever of unknown origin associated to inflammatory symptoms exposes the sagacity and the resolution capacity of the

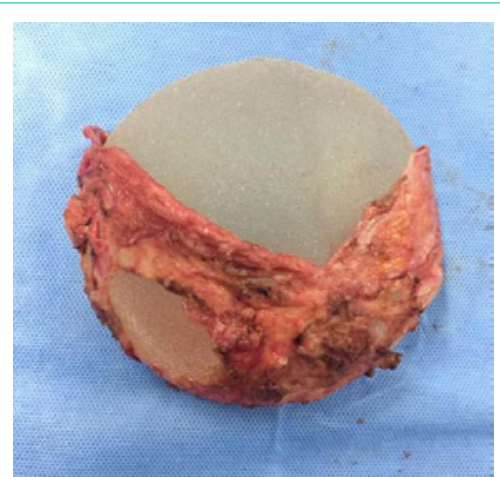

Figure 4: Capsulectomy left breast. The nodules located in the quadrant exceeded external and left armpit were resected.

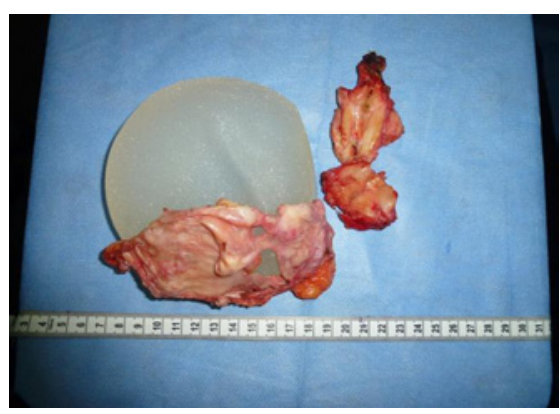

Figure 5: Prosthetics and right breast capsule.

doctor. In the submitted case, the only relevant background was the presence of silicone breast implants.

The age, sex and symptoms that this patient presented orientated the doctors to think on the first place about autoimmune disorders such as systemic lupus erythematosus or rheumatoid arthritis and subsequently in an oncohaematological case.

The laboratory exams, blood and urine tests, imaging studies and stool tests allowed the professionals to exclude an infectious process. This lead to categorize the diagnose as "Fever of unknown origin". Facing the lack of consolidated diagnoses, the need to include autoinflammatory process among them has to be contemplated in patients who were exposed for a long time to allergens like silicone.

Although there is a multicentric study which excluded the silicon as an inert material capable of producing an increase of CD8+ with a latency time of approximately 12 years [5], some published clinical trials revealed the association of rheumatological signs and symptoms in patients with silicone gel implants, highlighting among them rigidity, myalgia and sclerodermiform skin [6,7].

Facing this new situation, the immunological disorder of unknown origin and unexplained cause was attributed to the presence of silicone in the human body.

Several clinical pictures described by different authors associate the presence of silicone in the body as the triggering substance, as for example the ASIA syndrome (induced by adjuvants) or a variety of Still disease.

The ASIA syndrome was described for the first time by Schoenfeld 
in 2011. He recognizes the presence of silicone along with other three conditions as pathogenic to trigger the immune response of the individual.

The Still disease (adult variant of juvenile rheumatoid arthritis) is presented as an unknown cause entity whose classic presentation triad is fever, skin rashes and joint pain, associated to minor symptoms (lymph nodes, splenomegaly, serositis, abdominal pain) [8-10].

Due to the low prevalence or misdiagnose of this disorders, the casuistry is not significant enough, making it more difficult to establish an accurate diagnose of Still disease, therefore it is suggested to group the signs and symptoms in mayor and minor criteria to achieve an accurate diagnose. Two mayor and three minor criteria are required to confirm the diagnose [11] (Table 2).

Some cases have been reported in which a modulator of the immune response was enough to suppress it and revert the clinical condition of the patient using NSAIDs, glucocorticoids and immunosuppressant's with no need to remove the implants [12].

In opposition there are some who consider that the remission can only be reached by removing the allergen independently of the symptoms control achieved with medical treatment [13].

Even though the presented case complies with the totality of the proposed criteria, as much as major as minor, the initial treatment that lasted ten months including NSAIDs and glucocorticoids did not control the fever, that lead to a progressive deterioration of the patient's health, therefore the removal of the implants, capsules and siliconomas was the resolution made by the surgeons with satisfactory results in the immediate postoperative period (within the first 90 days) with the addition of $30 \mathrm{mg}$ per day. DE QUE?

\section{Conclusion}

The search for a diagnose and determination of the treatment in patients who consult for "fever of unknown origin" becomes a challenge for the professional and an uncertainty for the patients who are subjected to exhaustive clinical evaluations and complimentary exams to give a name to their condition.

The knowledge of the clinical background of the patient, its accurate interpretation and the correlation with the reason for consulting are the key point to reach a final diagnosis.
The silicone breast implants in women who consult for autoinflammatory symptoms and do not have a scientific verified diagnosis, should be consider as a probable cause until prove otherwise.

Future clinical trials evaluating a larger number of patients will reach definitive conclusions about the problem posed by this article with these patients.

\section{References}

1. Silverman BG, Brown SL, Bright RA, Kaczmarek RG, Arrowsmith-Lowe JB, Kessler DA. Reported complications of silicone gel breast implants: an epidemiologic review. Ann INtern Med. 1996; 124: 744-756.

2. Crétel E, Richard MA, Jean R, Durand JM. Still's like disease, breast prosthesis, and collagen implants. Rheumatol Int. 2001; 20: 129-131.

3. Israeli E, Agmon-Levin N, Blank M, Shoenfeld Y. Adjuvants and autoimmunity. Lupus. 2009; 18: 1217-1225.

4. Caldeira M, Caldeira A. Siliconosis: Autoimmune/Inflamatory Syndrome Induced by Adjuvants (ASIA) Imaj. 2012; 14: 137-138.

5. Hoshino T, Ohta A, Nakao M. TCR. T cells in peripheral blood of patients with adult still's disease. J Rheumatol. 1996; 23: 124-129.

6. Sanchez-Guerrero J, Colditz GA, Karlson EW, Hunter DJ, Speizer FE, Liang $\mathrm{MH}$, et al. Silicone breast implants and the risk of connective-tissue diseases and symptoms. N Engl J Med. 1995; 332: 1666-1670.

7. Hennekens $\mathrm{CH}$, Lee IM, Cook NR, Hebert PR, Karlson EW, LaMotte F, et al. Self-reported breast implants and connective-tissue diseases in female health professionals. A retrospective cohort study. JAMA. 1996; 275: 616621.

8. Le Lostec Z, Welker Y, Pauwels C. Maladie de Still de l'adulte dans les suites d'une pose de prothèse mammaire: una nouvelle cause de maladie de Still "rèactionnelle"? Rev Med Interne. 1998; 19: 474S.

9. Katayama I, Umeda T, Nishioka K. Adult Still's-disease like illness in a patient with silicone breast implants. Clin Rheumatol. 1998; 17: 81-82.

10. Shoenfeld Y, Agmon-Levin N. 'ASIA'-Autoimmune/inflammatory syndrome induced by adjuvants. J Autoimmun. 2011; 36: 1-3.

11. Bywaters EGL. Still's disease in the adult. Ann Rheum Dis. 1971; 30: 121133.

12. Ohta A, Yamaguchi M, Kaneoka H, Nagayoshi T, Hiida M. Adult Still's disease: review of 228 cases from the literature. J Rheumatol. 1987; 14 1139-1146.

13. Yamaguchi M, Ohta A, Tsunematsu T, Kasukawa R, Mizushima Y, Kashiwagi $\mathrm{H}$, et al. Preliminary criteria for classification of adult still's disease. J Rheumatol. 1992; 19: 424-430. 